Phosphorus and Sulfur, 1987, Vol. 30, pp. 349-352 Photocopying permitted by license only
(C) 1987 Gordon and Breach Science Publishers, Inc. Printed in the United Kingdom

\title{
ALKYLIDYNEPHOSPHINES - SYNTHESES AND REACTIVITY
}

\author{
G.BECKER*，W.BECKER，R.KNEBL，H.SCHMIDT，U.HILDENBRAND， AND \\ M.WESTERHAUSEN \\ Institut für Anorganische Chemie der Universität Stuttgart \\ Pfaffenwaldring 55, D-7000 Stuttgart 80
}

In the past, different methods have been utilized for the preparation of alkylidynephosphines. Whereas, however, small amounts of thermally instable derivatives might be obtained from reactions in the gas phase, the synthesis of phosphines which are stable under an inert atmosphere, as for instance those with a tert-butyl or a 1-adamantyl substituent at the carbon atom of the PEC group, is best started with tris(trimethylsilyl)phosphine itself or with the more reactive lithium bis(trimethylsilyl) phosphide.2 tetrahydrofuran complex. Treatment of either compound with acyl halides results in the formation of acylbis(trimethylsilyl)phosphines which, at room temperature, rearrange to the corresponding trimethylsilyl[1(trimethylsiloxy)alkylidene] isomers. As traces of hydrogen halide accelerate the conversion of tris(trimethylsilyl)phosphine to the triacyl derivatives, the use of the lithium phosphide is strongly recommended in all cases where impure acyl halides are used. In the presence of small amounts of solid sodium hydroxide suspended in an etherial solvent, the thus prepared trimethyl[1-(trimethylsiloxy)alkylidene] phosphines eliminate hexamethyldisiloxane to yield the required alkylidyne compounds. Running the decomposition without a solvent at a higher temperature, Regitz and coworkers were able to improve this method further.

After a careful spectroscopic characterization of both the methylidynephosphine and its tert-butyl derivative in particular, the reactivity of this class of compounds is now being studied in detail. Since the electronegativities of phosphorus (2.19) and carbon (2.55) are rather similar and differ substantially from the value of nitrogen (3.04), the question to be answered is whether alkylidynephosphines have more resemblance to alkines or nitriles with respect to their chemical properties. However, due to most results obtained thus far and discussed in the following, we are now justified in calling these compounds phosphaalkines.

Gier, Appel and others have already used the reaction between hydrogen chloride and various alkylidynephosphines in order to identify these phosphaalkines which had been formed only in small amounts. Halogens such as chlorine, bromine or iodine add stepwise to the P-C triple bond of 2,2-dimethylpropylidynephosphine to yield the (E) $/(Z)$-isomeric halogen(1-halogenalkylidene) compound first and the corresponding dihalogen $(1,1$-dihalogenalkyl) phosphine afterwards. 
Finally, with excess halogen the remaining weak $P-C$ single bond is cleaved and in addition to phosphorus(III) halide a 1,1,1-trihalogenalkane is formed. Remarkably, volatile halides of main group elements show a similar chemical behaviour towards alkylidynephosphines. In the reaction with germanium(IV) chloride, initially a mixture of $(E) /(Z)$-isomeric chloro[1-(trichlorogermyl)alkylidene]phosphine is formed; after the addition of a further amount of phosphaalkine a 1,2-diphosphetene (1) is obtained in a preparative scale finally and identified by $\mathrm{x}$-ray structure determination (eq. (1)).

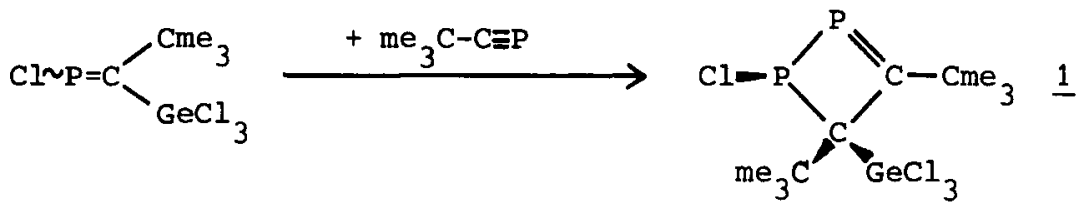

Although analogous intermediates have not yet been detected with phosphorus(III) bromide, the reaction route is expected to be very similar and compound 2 , the ultimately isolated 2,4,5-triphosphabicyclo-[1.1.1] pentane, might have been formed via an intramolecular addition of a $\mathrm{P}-\mathrm{Br}$ group to the $\mathrm{P}=\mathrm{C}$ bond of the cyclic system. The formation of only the $(E) /(Z)$-isomeric alkylidenephosphines 3 and 4 can be observed with $\operatorname{tin}($ IV) chloride and boron(III) bromide.
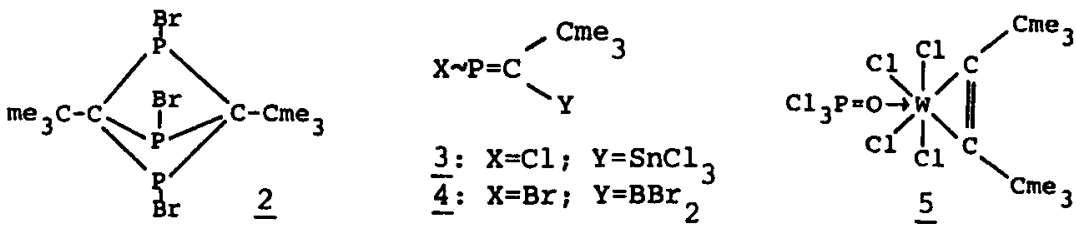

Whereas reactions between phosphaalkines and some common halides of main group elements are easy to perceive, the high oxydation power of several transition metal halides, on the contrary, gives rise to a scarcely understood formation of some very remarkable compounds. Similar to a metathesis, tungsten(VI) or molybdenum(V) chloride and 2,2-dimethylpropylidynephosphine react in phosphorus trichloride oxide to yield phosphorus(III) chloride and di(tert-butyl) acetylene complexes of tungsten(IV) (5) and molybdenum(III) chloride (6). From analogous reactions of tantalum(V) chloride rodshaped yellow crystals were isolated as main product (7) in addition to small amounts of the dark-red compound 8. The structures of both compounds are characterized by the presence of polycyclic systems and therefore suggest the possibility to oligomerize phosphaalkines in the presence of this type of transition metal halides. The bond type occurring in the previously unknown 1,3,5-triphosphatrishomocyclopropenylium cation of compound 8 , is best described by an overlapping of three p-orbitals at the phosphorus atoms which are directed to the centre of the six-membered ring (closed threecentre bond). With the solution of this structure the existence of a non-classical phosphenium ion has been proven for the first time. 


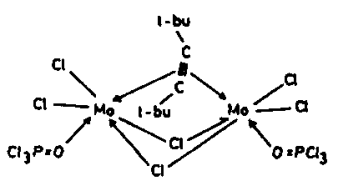

$\underline{6}$

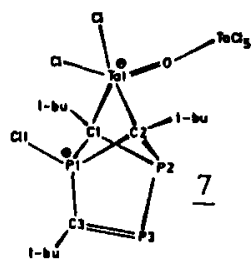

Alkylidynephosphines and alkines show a striking similarity with respect to their chemical reactivity not only to halides of main group elements, but also to complexes of transition metals in their low oxydation states. These investigations were first started by Nixon, Kroto and coworkers who in 1981 already published the synthesis of $\left[\eta^{2}-(2,2\right.$-dimethylpropylidynephosphine)]bis (triphenylphosphine)platinum prepared from the corresponding ethylene complex and the first thermally stable phosphaalkine. In the meantime, this research group has succeeded in the isolation of numerous further complexes with partially very complicated structures; nearly at the same time as Regitz et al., they reported the dimerization of 2,2dimethylpropylidynephosphine to a 1,3-diphosphacyclobutadiene bound to an $\left(n^{5}\right.$-cyclopentadienyl)cobalt fragment. In cooperation with Herrmann, Ziegler and coworkers, we were able to isolate the complexes $\underline{9}$ and 10 which have been formed via an addition of 2,2-dimethylpropylidynephosphine to the metal-metal multiple bonds of tetracarbonyl-di $\left(n^{5}\right.$-cyclopentadienyl)dimolybdenum and of $d i\left(\mu^{2}\right.$-carbonyl)di( $n^{5}$-pentamethylcyclopentadienyl) dirhodium. The fascinating

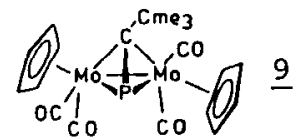

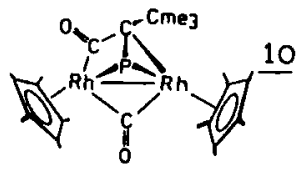

metathesis reactions with the metal-metal triple bond of hexa(tertbutoxy) ditungsten which had been carefully studied with alkines and nitriles by Schrock, Chisholm and coworkers, can be transferred to 2,2-dimethylpropylidynephosphine as well. However, the intermediates 11 and 12 which one would expect to have a $C-W$ or a $P-W$ triple bond, could not yet be detected; on the contrary, via an addition of excess phosphaalkine and a 1,3-shift of a tert-butoxy group from tungsten to phosphorus, 1-phospha-3-tungstena- (13) as well as $1 \lambda^{3}, 4 \lambda^{2}$-diphospha-3-tungstena-2,3-cyclobutadiene 14 are formed. Thus far we were able to isolate and characterize only the first mentioned compound, whereas the presence of the second one has been concluded from its nmr-spectra.

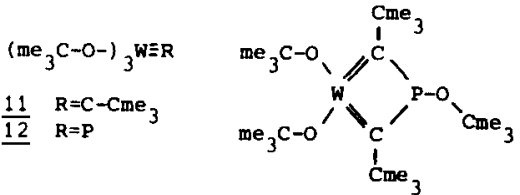

$\underline{13}$<smiles>COP1ON2P1C2(C)OC(C)(C)C</smiles>

$\underline{14}$ 
Already at room temperature organyl azides, nitrile oxides and diazoalkanes undergo fast [3+2]cycloaddition reactions with phosphaalkines to yield 1,2,3,4-triazaphospholes, 1,2,4-oxazaphospholes and 1H-1,2,4-diazaphospholes; Regitz and coworkers in particular, have applied these reactions in order to identify thermally instable alkylidynephosphines. Using kineticly stabilized cyclobutadienes, this research group also succeeded in the first preparation of $2 \lambda^{3}$-Dewar phosphinines; with cyclic 1,3-dienes $\lambda^{3}$-phosphinines can be obtained. In cooperation with Fluck and Neumüller who had prepared $1 \lambda^{5}, 3 \lambda^{5}$-diphosphacyclobutadiene, this compound was treated with 2,2-dimethylpropylidynephosphine to yield the first now fully characterized $1 \lambda^{3}, 3 \lambda^{5}, 5 \lambda^{5}$-triphosphabenzene.

Most probably an alkylidynephosphine might occur as an intermediate when ethyl benzoate and lithium bis(trimethylsilyl)phosphide. 2 tetrahydrofuran react to yield either the orange red lithium 1pheny 1-1,3-bis (trimethylsily1)-1,2-diphosphapropenide-3 1,2-dimethoxyethane (15) or the dark-green lithium 3,5-diphenyl-1,2-bis (trimethylsilyl)-1,2,4-triphosphacyclopent-3-en-5-ide - 3 1,2-dimethoxyethane (16). In this reaction lithium ethoxide and benzoylbis (trimethylsilyl) phosphine are expected to form first. Next, by exchanging a trimethylsilyl group versus lithium at the phosphorus atoms with excess lithium bis(trimethylsilyl)phosphide and by a subsequent elimination of lithium trimethylsilanolate, the acylphosphine or its alkylidene isomer can be transformed to phenylmethylidynephosphine. Finally, depending upon the applied conditions, the phosphaalkine and lithium bis(trimethylsilyl) phosphide which is still present or which might be newly produced from tris(trimethylsilyl)phosphine and lithium ethoxide, undergo addition reactions to yield compounds 15 and 16 .

Also the formation of lithium 3,5-di (tert-butyl)-1,2,4-triphosphacyclopentadienide.3 1,2-dimethoxyethane (17) from [2,2-dimethyl1-(trimethylsiloxy) propylidene]trimethylsilylphosphine and lithium bis(trimethylsilyl) phosphide.2 tetrahydrofuran can be expected to proceed in a similar manner; however, the surprising elimination of two trimethylsilyl groups has, as of the present, not been studied in detail.

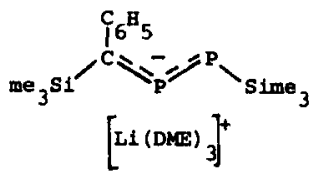

$\underline{15}$<smiles>[13CH3][13CH3]</smiles>

$\underline{16}$

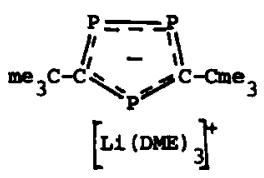

$\underline{17}$

For a detailed citation of literature see: G. Becker et al., Nova Acta Leopoldina, Neue Folge Nr. 264, 59 (1985) 55, as well as relevant abstracts of lectures of this meeting. 